\title{
DISTINCT DOPAMINERGIC ABNORMALITIES IN TRAUMATIC BRAIN INJURY AND PARKINSON'S DISEASE
}

Authors: Peter O Jenkins $\mathrm{PhD}^{1}$, Andreas-Antonios Roussakis $\mathrm{MD}^{2}$, Sara De Simoni $\mathrm{PhD}^{1}$, Niall Bourke MSc${ }^{1}$, Jessica Fleminger MEng ${ }^{1}$, James Cole $\mathrm{PhD}^{1}$, Paola Piccini $\mathrm{PhD}^{2}$, David J Sharp $\mathrm{PhD}^{1}$

\section{Affiliations:}

${ }^{1}$ Computational, Cognitive and Clinical Neuroimaging Laboratory, Imperial College London, Division of Brain Sciences, Hammersmith Campus, Imperial College London

${ }^{2}$ Neurology Imaging Unit, Centre of Neuroinflammation and Neurodegeneration, Division of Brain Sciences, Hammersmith Campus, Imperial College London

\section{Correspondence to:}

David J Sharp

The Computational, Cognitive and Clinical Neuroimaging Laboratory (C3NL)

Division of Brain Sciences, Department of Medicine

Imperial College London

Du Cane Road. W12 0NN

London, UK

david.sharp@imperial.ac.uk

Title character count: 86 , Number of references: 30 , Number of tables: 1 , Number of figures: 5, Word count abstract: 249, Word count paper: 3760

Running title: Dopaminergic abnormalities in TBI and PD

Key words: [165] Parkinson's disease/Parkinsonism; [123] SPECT; [264] Brain trauma

\section{Author Disclosures:}

Peter Jenkins reports no disclosures Andreas Roussakis reports no disclosures

Sara De Simoni reports no disclosures

Niall Bourke reports no disclosures

Jessica Fleminger reports no disclosures

James Cole reports no disclosures

Paola Piccini reports no disclosures

David Sharp reports no disclosures 
Study funding: This paper presents independent research funded by a National Institute of Health Research Professorship (NIHR-RP-011-048) awarded to DJS and supported by the NIHR CRF and Biomedical Research Centre at Imperial College Healthcare NHS Trust. The views expressed are those of the author(s) and not necessarily those of the NHS, the NIHR or the Department of Health. POJ is funded by Guarantors of Brain Clinical Fellowship. 


\section{ABSTRACT}

Objective: Traumatic brain injury (TBI) and REM behavioural disorder (RBD) are risk factors for Parkinson's disease (PD). Dopaminergic abnormalities are often seen after TBI, but patients usually lack parkinsonian features. We test whether TBI, PD and RBD have distinct striatal dopamine abnormalities using dopamine transporter imaging.

Methods: ${ }^{123}$ I-ioflupane SPECT scans were used in a cross-sectional study to measure dopamine transporter (DaT) levels in moderate/severe TBI, healthy controls, early PD and RBD patients. Caudate and putamen DaT, putamen-to-caudate ratios and left-right symmetry of DaT were compared.

Results: 108 participants (43 TBI, 26 PD, 8 RBD, 31 controls) were assessed. Early PD patients scored significantly higher on the UPDRS motor subscale than other groups. TBI and PD patients had reduced DaT levels in the caudate $(12.2 \%$ and $18.7 \%$ respectively) and putamen $(9.0 \%$ and $42.6 \%$ respectively) compared to controls. RBD patients had reduced DaT levels in the putamen $(12.8 \%)$ but not in the caudate compared to controls. PD and TBI patients showed distinct patterns of DaT reduction, with $\mathrm{PD}$ patients showing a lower putamen-to-caudate ratio. DaT asymmetry was greater in the PD group than other groups. 
Conclusions: The results show that early PD and TBI patients have distinct patterns of striatal dopamine abnormalities. Early PD and moderate/severe TBI patients showed similar reductions in caudate DaT binding, but PD patients showed a greater reduction in putamen DaT and a lower putamen-to-caudate ratio. The results suggest that parkinsonian motor signs are absent in these TBI patients because of relatively intact putaminal dopamine levels. 


\section{INTRODUCTION}

Traumatic brain injury (TBI) is a risk factor for the development of Parkinson's disease (PD).[1] Animal models of TBI produce a loss of dopaminergic fibres[2] and an associated hypodopaminergic state.[3] In humans, dopaminergic abnormalities are commonly seen in the striatum after moderate/severe TBI.[4-6] However, despite the presence of a hypodopaminergic state in many patients, it is rare that patients show parkinsonian features particularly after a single TBI.[6]

The lack of parkinsonian features after TBI may reflect the spatial pattern of dopaminergic abnormality. The striatum is a key target for dopaminergic projections from the midbrain dopaminergic nuclei. The striatum is anatomically sub-divided into the dorsal striatum (comprising most of the caudate and putamen) and the ventral striatum (comprising the nucleus accumbens, the ventromedial parts of the caudate and putamen and the striatal part of the olfactory tubercle).[7] The striatum can also be functionally segregated, with the posterior putamen involved in sensorimotor functions, the anterior striatum (i.e. caudate) supporting associative and cognitive functions and the ventral striatum associated with limbic functions.[8] Damage to distinct parts of the striatum can produce varying patterns of clinical features. For example, Parkinson's disease (PD) is characterised by the loss of dopaminergic fibres in a posterior to anterior progression in the striatum.[9] Early loss of dopaminergic input to the putamen is association with the development of motor signs, $[10,11]$ with around $30 \%$ of dopaminergic neurons lost in the putamen at symptom onset.[9] As dopaminergic cell loss advances anteriorly, cognitive impairments become apparent.[12] 
The dopamine transporter (DaT) is the main regulator of synaptic dopamine in the striatum and is down-regulated when dopamine levels are low.[13] ${ }^{123}$ I-ioflupane SPECT is widely used to assess dopamine transporter levels (DaT) in hypodopaminergic states[14, 15] and we have previously shown that DaT binding is reduced after TBI, particularly in the caudate.[6] This reduction is associated with cognitive impairment after TBI and we hypothesised that the absence of parkinsonian signs in patients with reduced striatal dopamine after TBI may be due to a subthreshold loss of dopaminergic input to the putamen.

Rapid-eye-movement sleep behaviour disorder (RBD) is also a risk factor for development of PD.[16] It is characterized by the loss of normal muscle atonia during the REM stage of sleep and patients show reduced striatal DaT levels, but to a lesser degree than that seen in PD.[17] Therefore, like TBI, RBD demonstrates abnormalities in the dopamine system but without the motor signs of Parkinson's disease for which it is also a known risk factor.

Here we use ${ }^{123}$ I-ioflupane SPECT to compare the density of striatal DaT between early PD, TBI, and RBD subjects as well as healthy controls. We investigate whether distinct spatial patterns of DaT abnormality are seen in these conditions and test whether TBI patients have less reduction in DaT within the putamen than PD patients to explain the lack of motor signs. 


\section{METHODS}

\section{Study design and participants}

A cross-sectional study of 43 TBI patients, 26 PD patients, 8 RBD patients and 31 controls was performed (Table 1). All participants had one ${ }^{123}$ I-ioflupane brain SPECT scan. 


\begin{tabular}{|c|c|c|c|c|}
\hline & $\begin{array}{c}\text { Controls } \\
\text { N=31 } \\
\text { Mean (SD) }\end{array}$ & $\begin{array}{c}\text { REM } \\
\text { Behavioural } \\
\text { Disorder } \\
\text { N=8 }\end{array}$ & $\begin{array}{l}\text { TBI Patients } \\
\qquad \mathbf{N}=43\end{array}$ & $\begin{array}{l}\text { PD patients } \\
\qquad \mathrm{N}=\mathbf{2 6}\end{array}$ \\
\hline Age & $46.8(14.7)$ & $68.0(6.8)$ & $39.7(12.1)$ & $58.0(9.0)$ \\
\hline Male sex - no. (\%) & $22(71)$ & $6(75)$ & $38(86)$ & $18(69)$ \\
\hline $\begin{array}{l}\text { Time since injury } \\
\text { (TBI)/symptom onset } \\
\text { (PD/RBD) - yrs }\end{array}$ & NA & $\begin{array}{c}5.28(3.22) \\
\text { Range[1.50,9.20] }\end{array}$ & $\begin{array}{c}5.94(7.21) \\
\text { Range[0.50,30.50] }\end{array}$ & $\begin{array}{c}1.90(1.54) \\
\text { Range[0.03,6.87] }\end{array}$ \\
\hline $\begin{array}{l}\text { UPDRS Part III Motor } \\
\text { subscale }\end{array}$ & NA & $2.1(1.1)$ & $3.6(9.3)$ & $25.2(9.3)$ \\
\hline Whole Striatum & $3.20(0.36)$ & $2.88(0.43)$ & $2.87(0.48)$ & $2.14(0.50)$ \\
\hline Striatum: Most affected side & $3.11(0.37)$ & $2.75(0.41)$ & $2.73(0.52)$ & $1.94(0.46)$ \\
\hline Striatum: Least affected side & $3.29(0.35)$ & $3.02(0.47)$ & $3.00(0.46)$ & $2.33(0.57)$ \\
\hline Striatal Asymmetry Index & $5.82(3.43)$ & $9.54(5.45)$ & $10.22(9.45)$ & $18.71(15.25)$ \\
\hline Whole Caudate & $3.39(0.42)$ & $3.20(0.71)$ & $2.97(0.49)$ & $2.76(0.63)$ \\
\hline Caudate: Most affected side & $3.26(0.44)$ & $3.09(0.72)$ & $2.80(0.52)$ & $2.55(0.58)$ \\
\hline Caudate: Least affected side & $3.51(0.42)$ & $3.30(0.70)$ & $3.14(0.50)$ & $2.96(0.70)$ \\
\hline Caudate Asymmetry Index & $7.67(6.02)$ & $7.01(5.04)$ & $11.69(8.87)$ & $15.13(10.78)$ \\
\hline Whole Putamen & $3.08(0.35)$ & $2.69(0.32)$ & $2.81(0.48)$ & $1.77(0.47)$ \\
\hline Putamen: Most affected side & $2.99(0.35)$ & $2.48(0.33)$ & $2.68(0.54)$ & $1.57(0.46)$ \\
\hline $\begin{array}{l}\text { Putamen: Least affected } \\
\text { side }\end{array}$ & $3.18(0.35)$ & $2.90(0.34)$ & $2.93(0.46)$ & $1.97(0.53)$ \\
\hline Putamen Asymmetry Index & $6.18(3.77)$ & $15.72(9.50)$ & $9.96(11.36)$ & $24.52(20.38)$ \\
\hline Putamen to caudate ratio & $0.91(0.07)$ & $0.86(0.15)$ & $0.95(0.06)$ & $0.65(0.10)$ \\
\hline
\end{tabular}

Table 1: Clinical characteristics and ${ }^{123}$ I-ioflupane SPECT results: Traumatic brain injury (TBI), Parkinson's disease (PD), REM Behavioural Disorder (RBD), Unified Parkinson's Disease Rating Scale (UPDRS), specific binding ratio (SBR).

TBI patients were recruited from specialist TBI clinics in London, UK. All TBI patients had a history of a single moderate-severe TBI based on Mayo criteria[18] at 
least 6 months prior to the study (see Supplementary File 1 for full demographic details). All TBI patients had subjective complaints of cognitive difficulties by participant, treating clinician, or caregiver. None had clinical evidence of motor signs of parkinsonism as assessed by a Neurologist. A screening visit involved medical history and physical examination including the Movement Disorder Society Unified Parkinson's disease rating scale (UPDRS) motor subscale.[19] TBI patients were excluded if taking a medication known to interfere with ${ }^{123}$ I-ioflupane binding.

PD patients were recruited from our Movement disorders clinic at the Imperial College Healthcare NHS Trust, London, UK. PD patients fulfilled the Queen Square Brain Bank Criteria for idiopathic PD. RBD patients were recruited from the sleep disorders clinic at the John Radcliffe Hospital, Oxford. The diagnosis of RBD was based on polysomnographic evidence according to standard International Classification of Sleep Disorders-II criteria by a consultant specializing in sleep disorders.[20] RBD was defined as an increase in tonic or phasic chin EMG activity during REM sleep and, either a history of elaborate motor activity associated with dream content, or the presence of behavioural manifestations occurring during REM sleep during polysomnographic recordings.[20] Patients were excluded if RBD was judged to be secondary to medication use, or was associated with other neurological conditions, including narcolepsy, PD, dementia or multiple system atrophy. RBD symptom duration was calculated as the time from the patient's defined symptom onset.

The three patient groups had differing ages (Table 1) and a group of healthy controls were recruited with an average age intermediate between the groups. Clinicians 
determining UPDRS motor subscale ratings were not blinded to the clinical diagnosis (i.e. group) at assessment.

\section{Standard Protocol Approvals, Registrations, and Patient Consents}

This study was reviewed and approved by the West London and GTAC Research Ethics Committee, UK and the Administration of Radioactive Substances Advisory Committee, UK. All participants provided written informed consent.

\section{Procedures}

Study procedures were carried out at Hammersmith Hospital, Imperial College London (London, UK) and Charing Cross Hospital (London, UK). PD patients were scanned in "off" dopaminergic medication state. Those participants on treatment for PD were asked to withdraw from medication 18 hours prior to the ${ }^{123}$ I-ioflupane SPECT scan. All PD patients were assessed for motor symptoms with the UPDRS part-III scale in "off" dopaminergic medication state.

Before administration of ${ }^{123}$ I-ioflupane, patients received potassium iodide tablets ( $2 \times 60 \mathrm{mg}$ ) to minimize radiation exposure to the thyroid gland. One hour later, a bolus intravenous injection of ${ }^{123} \mathrm{I}$-ioflupane (GE Healthcare Ltd) was administered to each individual (mean activity $185 \mathrm{MBq}$ ). SPECT images for all subjects were acquired using the same dual-headed gamma camera (Symbia T16, Siemens Healthcare) at 180 minutes post-injection with LEHR collimators, $128 \times 128$ matrix, 1.45 zoom, 128 projections, and 30 seconds per projection. 
To measure focal injury and exclude striatal damage TBI patients also had a T1weighted high-resolution MPRAGE scan (160 1-mm-thick transverse slices, $\mathrm{TR}=2300 \mathrm{~ms}, \mathrm{TE}=2.98 \mathrm{~ms}, \mathrm{FA}=9^{\circ}$, in-plane resolution $=1 \times 1 \mathrm{~mm}$, matrix size $=256 \times 256$, field of view $=25.6 \times 25.6 \mathrm{~cm}$ ). Analysis was repeated with TBI patients with lesions in the caudate or putamen removed.

\section{${ }^{123}$ I-ioflupane Single Photon Emission Computed Tomography analysis}

A semi-quantification analysis approach for each individual was used to quantify reconstructed tomographic data. Acquired data were anonymised and then reconstructed with an ordered subset expectation maximization (OSEM) based iterative algorithm (HybridRecon, HERMES Medical Solutions; Stockholm, Sweden) including corrections for attenuation, scatter, and resolution. The reconstructed data were analysed using BRASS software (HERMES Medical Solutions). The software uses automatic image registration to align the participant's image to a template. Within the template are a series of predefined volumes of interest that can be applied to the image being analysed. Binding ratios were extracted automatically for each volume of interest and once complete, automatic fitting was assessed visually for each individual scan by two experienced raters as a standard quality control check. During quality control, all images were inspected and minimal adjustments involving shifting, rotating but not scaling, were applied to only a few scans where the outline of the template was not capturing well the activity within the occipital lobe. The raters were not blinded to the clinical diagnosis during the quality control check.

Specific-to-nonspecific binding ratios (SBR) were measured for each caudate and putamen relative to nonspecific binding measured from the occipital cortex. The SBR is defined as: ([region of interest counts - background counts]/background counts), 
where the occipital cortex is assumed to be devoid of DaT. The specific DaT binding as reflected by the ${ }^{123}$ I-ioflupane SBR was calculated for each caudate and putamen in both hemispheres. The average caudate and putamen SBR was calculated per individual as the mean uptake value for both hemispheres. To assess the asymmetry between the caudal and putaminal SBR in each hemisphere the asymmetry index was calculated using the following formula: $[(\mathrm{X}-\mathrm{Y}) /(\mathrm{X}+\mathrm{Y})] \times 2 \times 100 ; \mathrm{X}=$ least affected side, $\mathrm{Y}=$ most affected side.[21]

\section{Statistical analyses}

Statistical analysis and graph illustration were performed using R (http://www.Rproject.org/). Group characteristics were compared using independent sample t-tests (for age) and Fisher's exact test (gender).

To assess the effect of group (i.e. TBI, PD, RBD or control) on caudate and putamen SBR and caudate and putamen asymmetry indices, linear mixed effects models were used with group and region defined as fixed effects, whereas subject was defined as a random effect to model variability in subject intercepts. Age was included as a covariate. Post-hoc unpaired sample t-tests were used to investigate any significant main effects or interactions. A two-sided p-value of 0.05 was considered to indicate statistical significance.

To assess the effect of group on putamen-to-caudate ratio we used linear regression to predict ratio with group and age as explanatory variables. Post-hoc unpaired sample ttests were used to investigate any group effect. 


\section{Data availability}

Anonymized data will be shared on request through personal correspondence.

\section{RESULTS}

\section{Clinical characteristics}

Clinical characteristics and SPECT scan results are summarised in Table 1, with example SPECT scans shown in Figure 1. The PD and RBD patients were older than the TBI patient group as would be expected for these conditions (mean ages \pm standard deviation; $58.0 \pm 9.0,68.0 \pm 6.8$ and $39.7 \pm 12.1$ respectively; $\mathrm{p}<0.001$ for difference in age between both PD and RBD patients and TBI patients). The control group were intermediate in age $(46.8 \pm 14.7)$. There was a male preponderance in all groups (Percentage males: TBI 86\%, PD 69\%, RBD 75\%, Controls 71\%). PD patients were relatively newly diagnosed, reflected in the average time between symptom onset and scan (1.90 \pm 1.54 years). TBI patients were all in the chronic phase (>6 months from injury) with average time since injury 5.94 years (range 0.5 to 30.5 years). PD patients had the highest UPDRS motor subscale score $(25.2 \pm 9.3, \mathrm{p}<0.001$ compared to both TBI and RBD) with both the RBD $(2.1 \pm 1.1)$ and TBI $(3.6 \pm 9.3)$ groups having much lower values, reflecting the absence of Parkinsonian features. 
Traumatic brain injury and Parkinson's disease produce reduced ${ }^{123}$ IIoflupane specific binding ratios in the caudate and putamen

Linear mixed effects modelling showed a significant interaction between group and region $(F(3,104)=44.1, p<0.001)$, the result of distinct patterns of ${ }^{123}$ I-Ioflupane DaT across the caudate and putamen. Post hoc t-tests showed that both TBI patients and PD patients had reduced caudate DaT compared to controls $(\mathrm{t}(69.7)=-3.88, \mathrm{p}<0.001$ and $\mathrm{t}(42.4)=-4.37, \mathrm{p}<0.001$ respectively). TBI and PD showed similarly low caudate $\operatorname{DaT}(\mathrm{p}=0.14)$ whereas RBD patients showed no differences to the other groups (all $\mathrm{p}$ values >0.1) (Table 1 and Figure 2A). In the putamen, PD patients showed marked reductions in DaT compared to controls, as well as TBI and RBD patients $(\mathrm{t}(45.2)=-$ 11.79, $\mathrm{p}<0.001, \mathrm{t}(54.0)=-8.78, \mathrm{p}<0.001$ and $\mathrm{t}(17.4)=-6.30, \mathrm{p}<0.001$ respectively $)$ TBI patients also showed reduced putamen DaT compared to controls but no difference with the RBD group $(\mathrm{t}(72)=-2.86, \mathrm{p}=0.006$ and $\mathrm{p}=0.39$ respectively). RBD patients had significantly reduced putamen SBR compared to controls $(\mathrm{t}(11.7)=-3.07, \mathrm{p}=0.01)$.

The largest effect on DaT was seen for PD patients in the putamen (Cohen's d=3.14 [95\% confidence interval CI] $[2.34,3.93] ; 42.6 \%$ reduction compared to controls). TBI and RBD patients showed lesser effects in the putamen (Cohen's $d=0.67[0.19$, $1.16]$ and $1.22[0.37,2.07] ; 9.0 \%$ and $12.8 \%$ reductions, respectively). In the caudate effect sizes for PD and TBI patients were similar (Cohen's $d=1.16[0.59,1.74]$ and $0.91[0.42,1.41] ; 18.7 \%$ and $12.2 \%$ reductions, respectively). RBD patients showed the smallest effect in the caudate (Cohen's $\mathrm{d}=0.29[-0.51,1.1], 5.7 \%$ reduction) (Figure 2B). 


\section{Traumatic brain injury and Parkinson's disease produce differing patterns of striatal SBR changes}

To explore whether TBI, RBD and PD affect the putamen and caudate to differing degrees we analysed the putamen-to-caudate ratios across groups (Figure 3). Linear modelling showed a significant effect of group $(F(3,103)=78.34, p<0.001)$. Post hoc tests revealed that the PD group had significantly lower putamen-to-caudate ratios than controls, TBI patients and RBD patients $(\mathrm{t}(43.7)=-11.36, \mathrm{p}<0.001, \mathrm{t}(35.7)=-$ 13.69, $\mathrm{p}<0.001$ and $\mathrm{t}(9.1)=-3.88, \mathrm{p}=0.004$ respectively). The TBI group showed an increase in the putamen-to-caudate ratio compared to controls of borderline significance $(\mathrm{t}(57.8)=1.98, \mathrm{p}=0.053)$ but no difference to $\mathrm{RBD}$ patients $(\mathrm{t}(7.4)=1.52$, $\mathrm{p}=0.17)$. The RBD group was not different to the controls $(\mathrm{t}(7.8)=-0.93, \mathrm{p}=0.38)$.

\section{Parkinson's disease patients show asymmetric reduction in specific binding ratios}

We next investigate whether ${ }^{123}$ I-Ioflupane SBR was asymmetrical. A linear mixed effects model showed a significant interaction between group and region for the asymmetry indices $(F(3,104)=7.09, p<0.001)$. In the caudate, post hoc tests revealed the asymmetry index was significantly greater for the PD patients compared to control and $\mathrm{RBD}$ groups $(\mathrm{t}(37.7)=3.14, \mathrm{p}=0.003$ and $\mathrm{t}(26.1)=2.93, \mathrm{p}=0.007$ respectively). TBI patients also showed increased caudate asymmetry compared to controls $(\mathrm{t}(71.8)=2.32, \mathrm{p}=0.02)$ and a borderline increase compared to the RBD group $(t(16.5)=2.09, p=0.053)$. PD and TBI group were not different $(p=0.18)$ and there was no difference between the RBD and controls ( $\mathrm{p}=0.76$ ) (Figure 4). 
In the putamen, PD patients showed increased asymmetry indices compared to both the control and TBI groups $(\mathrm{t}(26.4)=4.52, \mathrm{p}<0.001$ and $\mathrm{t}(34.6)=3.34, \mathrm{p}=0.002$ respectively), but not compared to the RBD group ( $\mathrm{p}=0.10)$. The TBI patients had increased asymmetry indices compared to the controls $(\mathrm{t}(54.0)=2.03, \mathrm{p}=0.047)$ but not the RBD patients $(p=0.16)$. RBD patients had an increased asymmetry index compared to the controls $(\mathrm{t}(7.58)=2.79, \mathrm{p}=0.02)$. PD patients showed a greater asymmetry in the putamen compared to caudate $(\mathrm{t}(25)=3.09, \mathrm{p}=0.005)$. Asymmetry indices in the other groups were not different between the regions $(\mathrm{p}>0.1)$.

No effect on results if TBI patients with striatal lesions removed from the analysis

Macroscopic striatal lesions visible on T1 MRI were uncommon in the TBI group (Figure 5). We have previously shown that frontal lesion size is not correlated with DaT binding and nor is motor UPDRS scores in TBI patients.[6] Six patients had visible lesions in their caudate and three in the putamen. When present, these lesions were small; in the caudate the mean size was $5.4 \%$ of total caudate volume (range 0.4 to $10.4 \%$ ) and in the putamen just $2.4 \%$ (range 0.7 to $5.7 \%$ ). When the above analyses were repeated with TBI patients removed if they had a lesion present, all results above remained qualitatively similar except for the borderline difference in putamen asymmetry index between TBI patients and controls (all TBI patients included: $\mathrm{t}(54.0)=2.03, \mathrm{p}=0.047$; TBI patients with lesions excluded: $\mathrm{t}(49.8)=1.78, \mathrm{p}=0.08)$. No patients had macroscopic focal lesions in the substantia nigra or along the nigrostriatal tract. 


\section{DISCUSSION}

Our findings show that moderate/severe TBI and early PD can both lead to reduced dopamine levels in the caudate and putamen as measured by ${ }^{123}$ I-ioflupane SPECT. Reductions in the dopamine transporter (DaT) in the caudate were similar between our moderate/severe TBI and PD groups. However, reductions in putaminal DaT were much more severe in early PD than TBI. The posterior-to-anterior gradient of dopamine loss in PD was reflected in lower putamen-to-caudate DaT binding ratios compared to both TBI and RBD patients. Although motor impairments are common after moderate/severe TBI, parkinsonian features are relatively infrequent.[6] The distinct patterns of striatal DaT abnormality seen in TBI and in early PD patients may account for the absence of motor parkinsonism in TBI patients.

DaT abnormalities are commonly seen following TBI and superficially similar to those seen in PD, being frequently reported as suggesting Parkinson's disease in clinical reports.[6] Therefore, the absence of parkinsonian features in these patients appears surprising. Our direct comparison between PD and TBI provides an explanation for this apparent paradox. Firstly, DaT binding within the putamen after TBI is not reduced to the level thought necessary to produce motor parkinsonism. Previous studies have shown that DaT reductions in the putamen of around $50 \%$ are associated with the presence of motor symptoms in PD.[9] Our findings are in keeping with this observation as our early PD patients showed a $43 \%$ reduction in putamen DaT binding. In contrast, TBI patients showed only a $9 \%$ reduction in putamen DaT binding. In contrast, DaT binding in the caudate was reduced to similar levels in TBI and early PD. Caudate DaT levels have been linked to cognitive 
performance in PD patients[12] and we have previously shown that caudate DaT levels relate to cognitive impairments post TBI.[6] Therefore, reduced dopamine in the caudate after TBI may contribute to cognitive impairments after TBI, even in the absence of motor abnormalities.

Our results have a number of important clinical implications. Although ${ }^{123}$ I-ioflupane SPECT scans are not routinely performed following TBI, around a third of patients will have abnormalities following moderate/severe TBI.[6] This information can clarify the cause of post-traumatic problems, even in the absence of motor abnormalities. For example, it can be unclear why patients have cognitive and behavioural abnormalities after TBI. Hence, the identification of a hypodopaminergic state may clarify the mechanism for their clinical presentation.[22] Information about post-traumatic striatal dopamine levels can also guide treatment choices. For example, cognitive enhancers are often used in patients with cognitive impairment and many act through the dopamine neurotransmitter system. We have recently shown that response to methylphenidate, which is widely used following TBI, can be predicted if a patient's dopaminergic state is known, demonstrating the clinical value of this diagnostic information.[23] From the perspective of patients with possible Parkinson's disease, it is also important to appreciate that DaT abnormalities may be due to a remote history of traumatic brain injury, which may not previously have been thought relevant. The differing patterns of DaT abnormalities in PD and after a TBI may help to clarify the cause of the current symptoms. The quantitative analysis techniques we used are already commonly employed on clinical scanners and so information on DaT binding in the caudate and putamen is readily available to clinicians to aid the interpretation of any DaT abnormalities. Hence, ${ }^{123}$ I-ioflupane 
SPECT scanning provides a straightforward way to obtain additional clinically relevant information for the assessment of TBI.

TBI is a risk factor for future development of PD.[1] The accumulation of $\alpha$-synuclein is a key pathological hallmark of $\mathrm{PD}[24]$ and the development of PD after TBI is dependent on genetic variability in the $\alpha$-synuclein gene,[25] which regulates $\alpha$ synuclein production. TBI leads to increased levels of $\alpha$-synuclein,[26] which could accelerate the development of PD in susceptible individuals. Reductions in striatal dopamine might also predispose patients to an earlier onset of PD, as a critical threshold for parkinsonian symptoms will be reached sooner. Twin studies support accelerated disease onset after TBI. In twins who are both affected by PD, the twin with a history of TBI is more likely to have an earlier onset of disease.[27] TBI could also trigger a neurodegenerative process that ends in PD, perhaps through continued over-production of $\alpha$-synuclein and accelerated loss of dopaminergic neurons. Hence, rather than a static 'hit' to the dopaminergic system, TBI could instead produce accelerated degeneration of the dopaminergic fibres.

Reductions in striatal DaT binding following a TBI could be due to structural damage to the dopaminergic terminals in the striatum, functional down-regulation of DaT expression, damage to the dopaminergic cell bodies in the brain stem or disruption of the ascending nigrostriatal tracts. Macroscopic striatal lesions visible on T1 MRI in the TBI group were uncommon and when present, very small. Removal of patients with visible lesions did not change the main results, i.e. reduced caudate and putamen DaT levels in TBI patients compared to controls and significantly lower putamen-tocaudate ratios between PD and TBI patients. No patients had macroscopic focal lesions in the substantia nigra or along the nigro-striatal tract. Previously, we have 
shown that reduced volume of the substantia nigra, where dopaminergic cell bodies reside in the brain stem, correlate with striatal binding.[6] In addition, we showed evidence of microstructural damage in the area through which the ascending dopaminergic fibres pass to the caudate. Taken together these findings suggest that damage to the nigrostriatal system is likely to be a key determinant of reduced striatal DaT levels after TBI.[6]

REM behavioural disorder is also a risk factor for the development of PD. RBD and TBI patients showed similar putaminal DaT reductions (13\% and 9\%). Neither group showed motor parkinsonism although DaT was significantly reduced compared to controls, in keeping with a sub-threshold reduction of putaminal dopamine. These results are in keeping with a recent meta-analysis of presynaptic dopaminergic neuroimaging that showed a progressive reduction in uptake in the putamen between the control, RBD and PD groups.[28] Our RBD group did not show reduced levels of caudate DaT, in contrast to the meta-analysis, which also reported a significant decrease in caudate SBR in RBD patients compared to controls. Our null result for RBD in the caudate may have been related to the relatively small sample size for the RBD group (8 compared to 43 and 26, respectively).

Our study has a number of limitations. Our study groups were not matched for age. However, this discrepancy reflects the general ages of the disease populations studied and is unlikely to have biased our main findings. The reduction in DaT following TBI is seen despite these patients being younger on average than the PD group, making the similarity in caudate DaT levels across the groups more striking. In addition, clinicians assessing patients, in particular the UPDRS motor subscale, were not blinded to the clinical diagnosis (i.e. TBI, PD or RBD). This has the potential to bias 
the reported values for the UPDRS. We also only report cross-sectional data, which does not allow differentiation between alternative explanations for the link between PD and TBI. Longitudinal studies using dopaminergic imaging could clarify whether there is static or accelerating loss of striatal dopamine after TBI. Finally, our in vivo imaging assessment uses a semi-quantitative technique, which assumes that non-

specific uptake of ${ }^{123}$ I-ioflupane is equivalent throughout the brain. If TBI caused variable changes to this non-specific uptake in different brain regions, this could bias the results. We have no reason to suspect distinct non-specific uptake across the striatum to explain the pattern of abnormalities we observed and our results are also in line with animal models of TBI using different techniques, which also show reduced DaT expression in the striatum.[3, 29, 30]

In conclusion, TBI leads to loss of striatal dopamine as measured by ${ }^{123}$ I-ioflupane SPECT. Much greater reductions of putaminal DaT are seen in early PD, explaining why motor signs of parkinsonism are not commonly seen following TBI. In addition, TBI patients do not show the posterior-to-anterior gradient of DaT loss seen in early PD and also RBD, another risk factor for developing PD. The work informs the link between TBI and PD, but longitudinal studies will be needed to determine whether a static reduction in striatal dopamine or a progressive neurodegenerative process is triggered by TBI.

\section{ACKNOWLEDGMENTS:}

DJS was funded by a National Institute of Health Research Professorship (NIHR-RP011-048). POJ is funded by Guarantors of Brain Clinical Fellowship. PP received 
funding from the Michael J Fox Foundation for Parkinson's Research. The research was also supported by the National Institute for Health Research (NIHR) Imperial Biomedical Research Centre.

The authors would also like to thank Associate Professor Michele $\mathrm{Hu}$, Nuffield Department of Clinical Neurosciences, University of Oxford for her help recruiting the RBD patients.

\section{AUTHORS' ROLES:}

PJ, AR, SD, PP, DS contributed to the conception and design of the study. PJ, AR, SD, NB, JF, JC, PP, DS all contributed to the acquisition and analysis of the data. PJ, AR, PP and DS were responsible for the bulk of drafting the manuscript

\section{FINANCIAL DISCLOSURES FOR ALL AUTHORS:}

None 


\section{FIGURES:}

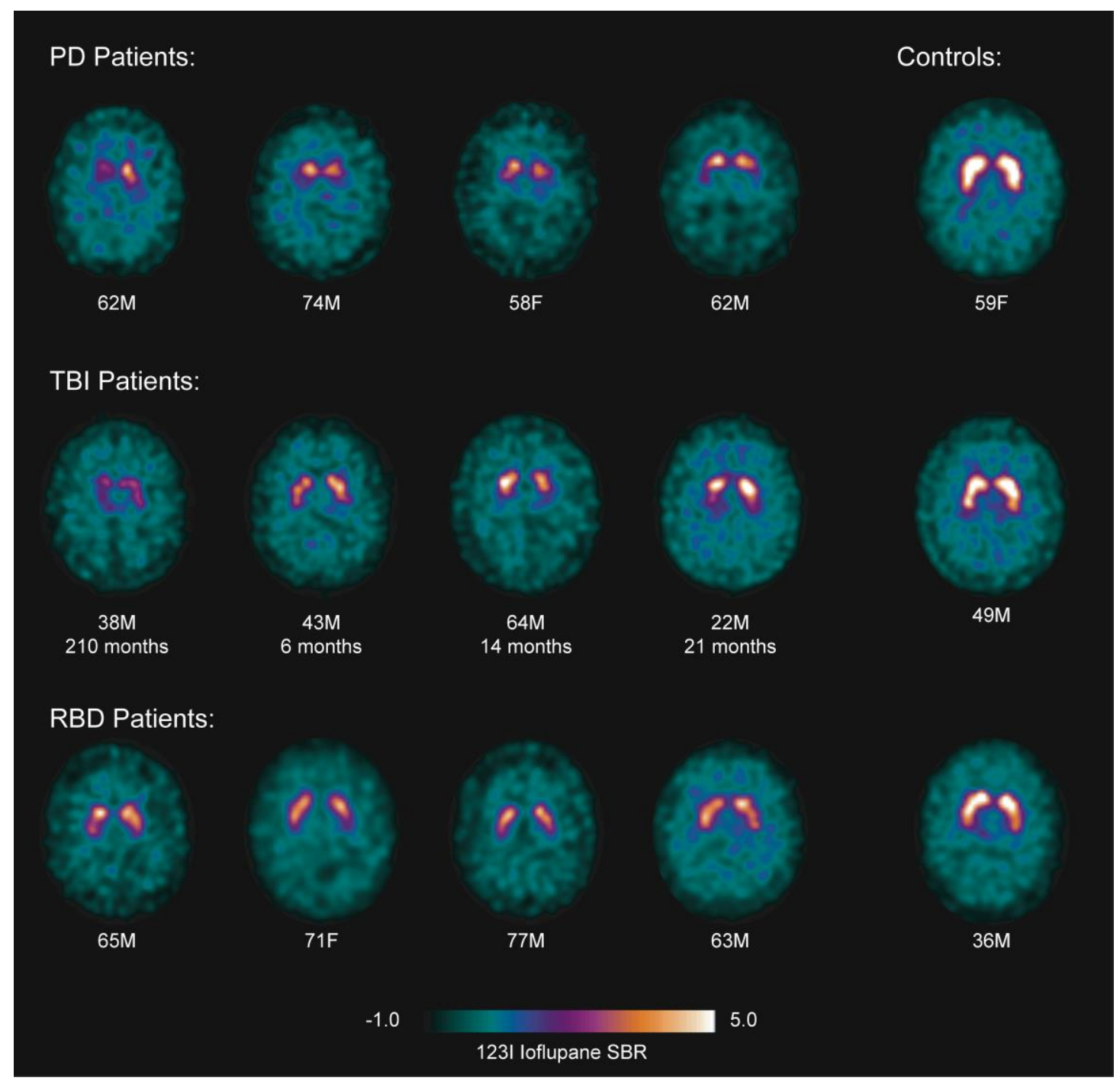

Figure 1. ${ }^{123}$ I-ioflupane SPECT scan examples: A selection of 4 PD, TBI and RBD patients and 3 controls. Subject's age, gender $(\mathrm{M} / \mathrm{F})$ and the time since injury (TBI)/symptom onset (PD and RBD) in years is shown. The colour scale shows the ${ }^{123}$ I-ioflupane SBR. 

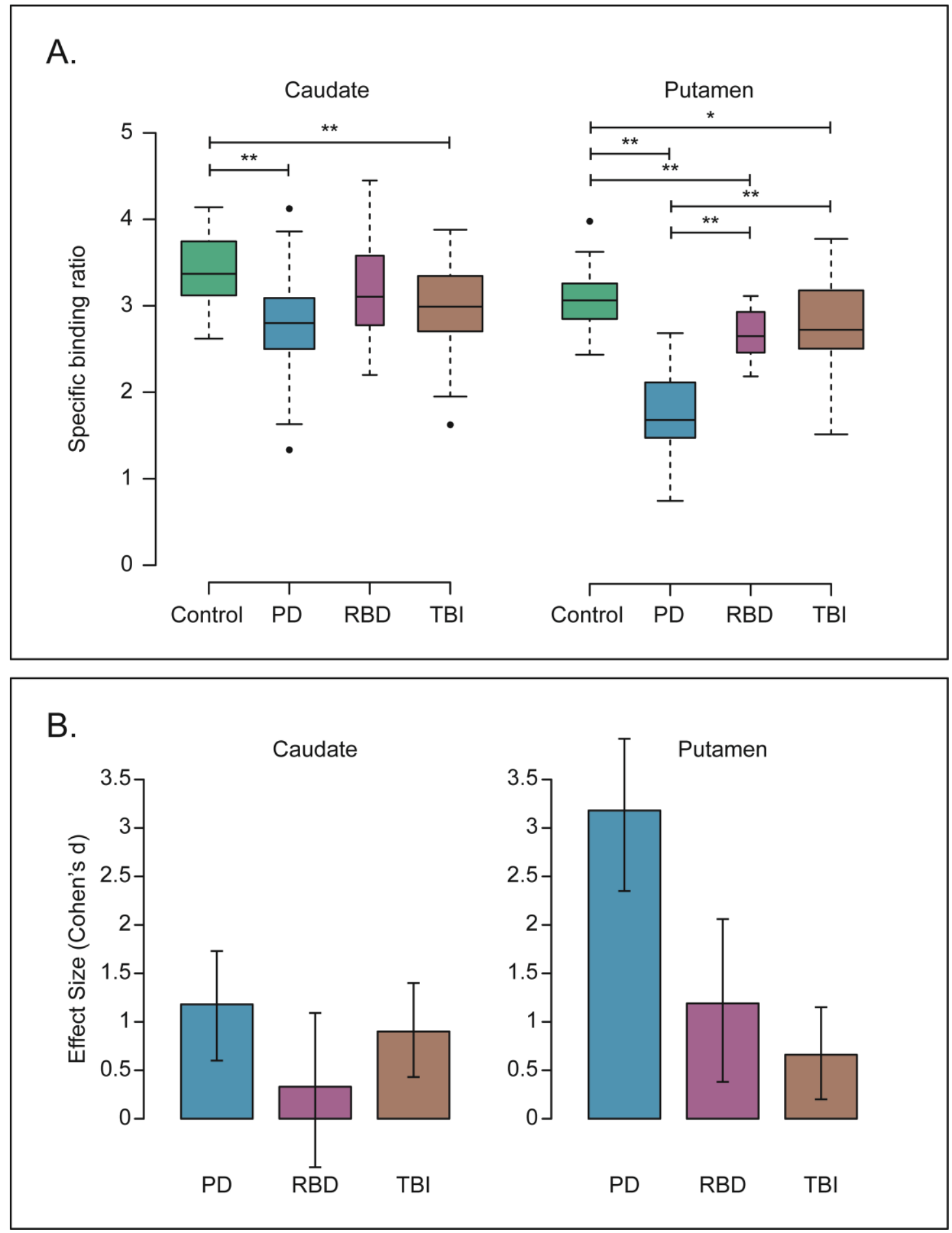

Figure 2. ${ }^{123}$ I-ioflupane SPECT scan results: A. Specific binding ratios in the caudate and putamen for the healthy controls (Green), Parkinson's disease (PD) (Blue), REM behavioural disorder (RBD) (Magenta) and traumatic brain injury (TBI) (Brown) patients. The width of the boxplots is proportional to the group sizes. * denotes $\mathrm{p}<0.05$, $* *$ denotes $\mathrm{p}<0.01$. B. Effect sizes (Cohen's d) in the caudate and 
putamen for PD, RBD and TBI patients compared to controls. Error bars represent $95 \%$ confidence intervals.

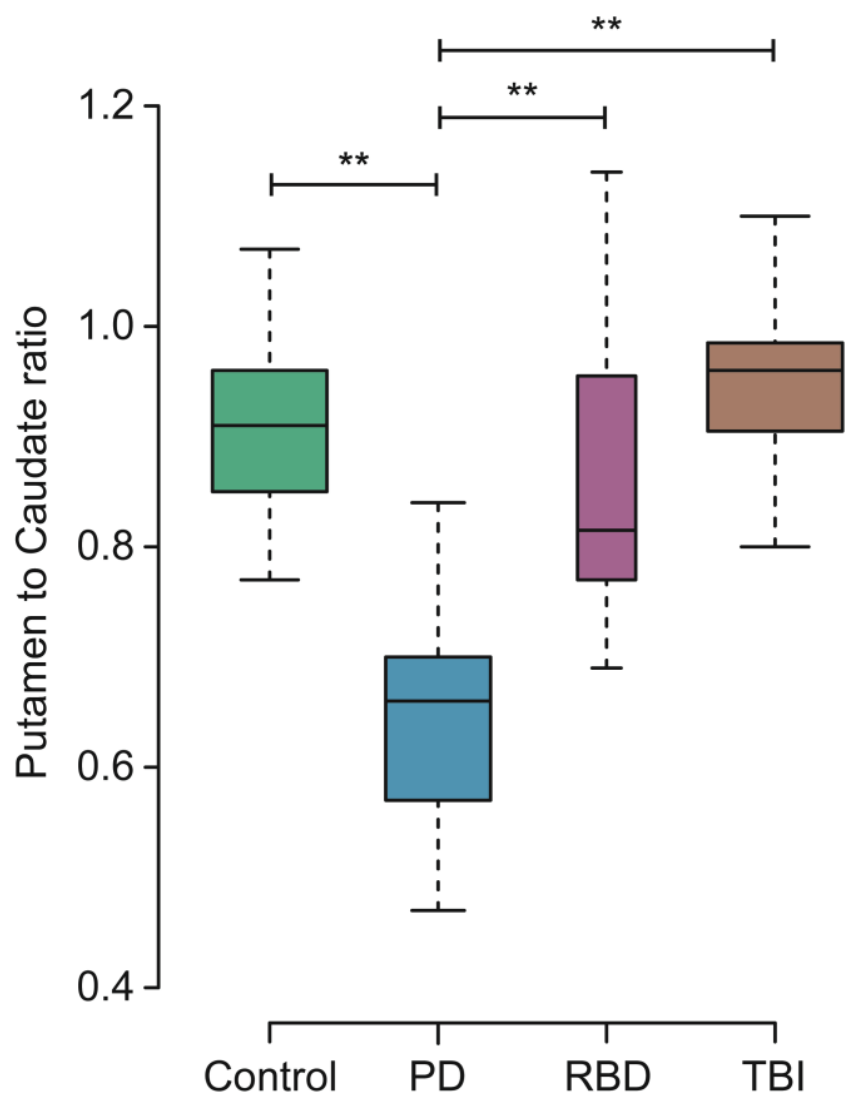

Figure 3. Putamen-to-caudate ratios across groups: The width of the boxplots is proportional to the group sizes. $* *$ denotes $\mathrm{p}<0.01$ 


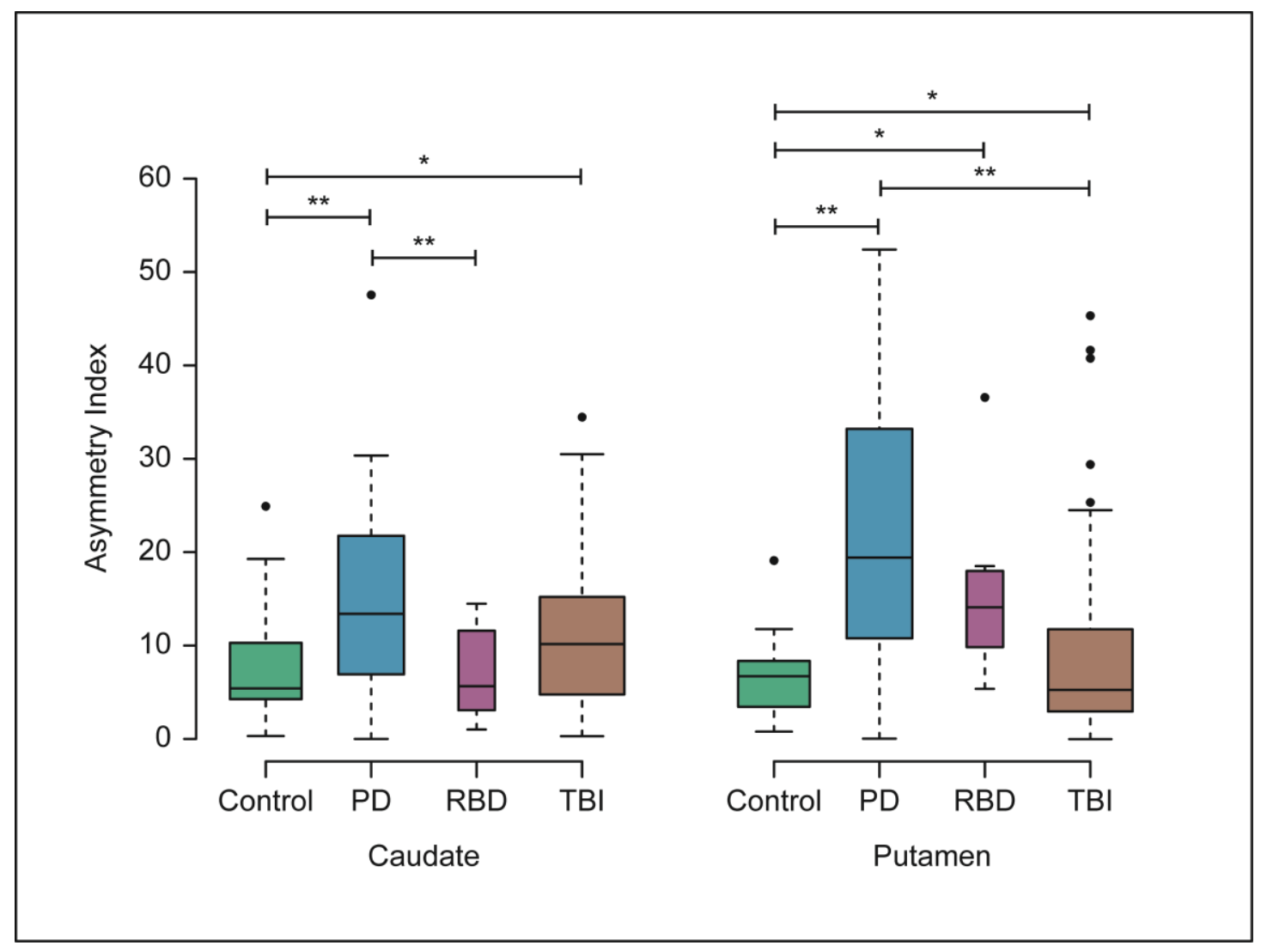

Figure 4. Asymmetry indices in the caudate and putamen: Asymmetry indices in the caudate and putamen for the healthy controls (Green), Parkinson's disease (PD) (Blue), REM behavioural disorder (RBD) (Magenta) and traumatic brain injury (TBI) (Brown) patients. The width of the boxplots is proportional to the group sizes. . * denotes $\mathrm{p}<0.05, * *$ denotes $\mathrm{p}<0.01$. 


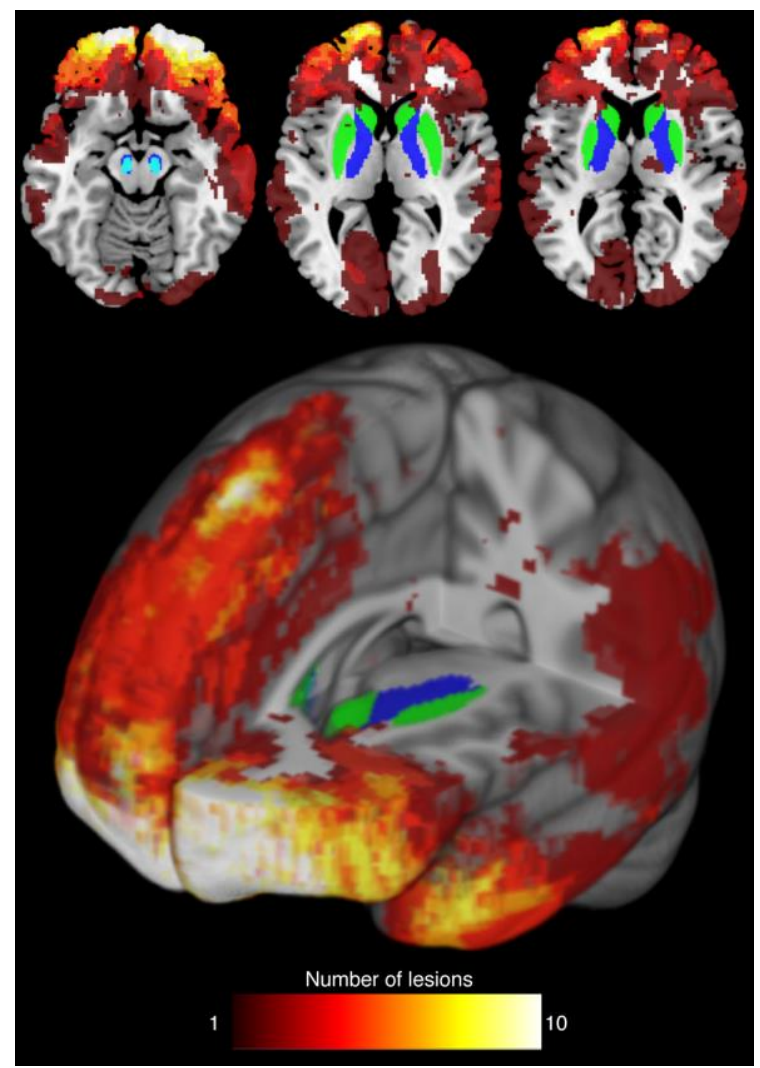

Figure 5. Lesion map: Thirty patients had focal lesions visible on T1 MRI. These were overlaid to produce a lesion map. Maximal areas of overlap of lesions were seen in the anterior frontal and temporal lobes. There were no lesions in the substantia nigra (light blue) and minimal lesion load in the striatum (green) and in the area through which the nigrostriatal tract passes from the substantia nigra to the striatum (dark blue). (From[6])

\section{REFERENCES}

1 Gardner RC, Burke JF, Nettiksimmons J, et al. Traumatic brain injury in later life increases risk for Parkinson disease. Ann Neurol 2015;77:987-95.

2 van Bregt DR, Thomas TC, Hinzman JM, et al. Substantia nigra vulnerability after a single moderate diffuse brain injury in the rat. Exp Neurol 2012;234:8-19. 
3 Wagner AK, Sokoloski JE, Ren D, et al. Controlled cortical impact injury affects dopaminergic transmission in the rat striatum. J Neurochem 2005;95:457-65. 4 Donnemiller E, Brenneis C, Wissel J, et al. Impaired dopaminergic neurotransmission in patients with traumatic brain injury: a SPECT study using 123Ibeta-CIT and 123I-IBZM. Eur J Nucl Med 2000;27:1410-4.

5 Wagner AK, Scanlon JM, Becker CR, et al. The influence of genetic variants on striatal dopamine transporter and D2 receptor binding after TBI. J Cereb Blood Flow Metab 2014;34:1328-39.

6 Jenkins PO, De Simoni S, Bourke NJ, et al. Dopaminergic abnormalities following traumatic brain injury. Brain 2018.

7 Joel D, Weiner I. The connections of the dopaminergic system with the striatum in rats and primates: an analysis with respect to the functional and compartmental organization of the striatum. Neuroscience 2000;96:451-74. 8 Redgrave P, Rodriguez M, Smith Y, et al. Goal-directed and habitual control in the basal ganglia: implications for Parkinson's disease. Nat Rev Neurosci 2010;11:760-72.

9 Cheng HC, Ulane CM, Burke RE. Clinical progression in Parkinson disease and the neurobiology of axons. Ann Neurol 2010;67:715-25.

10 Guttman M, Burkholder J, Kish SJ, et al. [11C]RTI-32 PET studies of the dopamine transporter in early dopa-naive Parkinson's disease: implications for the symptomatic threshold. Neurology 1997;48:1578-83.

11 Ma Y, Dhawan V, Mentis M, et al. Parametric mapping of [18F]FPCIT binding in early stage Parkinson's disease: a PET study. Synapse 2002;45:125-33. 
12 Ekman U, Eriksson J, Forsgren L, et al. Functional brain activity and presynaptic dopamine uptake in patients with Parkinson's disease and mild cognitive impairment: a cross-sectional study. Lancet Neurol 2012;11:679-87.

13 Gordon I, Weizman R, Rehavi M. Modulatory effect of agents active in the presynaptic dopaminergic system on the striatal dopamine transporter. Eur J Pharmacol 1996;298:27-30.

14 Catafau AM, Tolosa E, Da TCUPSSG. Impact of dopamine transporter SPECT using 123I-Ioflupane on diagnosis and management of patients with clinically uncertain Parkinsonian syndromes. Mov Disord 2004;19:1175-82.

15 Kupsch AR, Bajaj N, Weiland F, et al. Impact of DaTscan SPECT imaging on clinical management, diagnosis, confidence of diagnosis, quality of life, health resource use and safety in patients with clinically uncertain parkinsonian syndromes: a prospective 1-year follow-up of an open-label controlled study. J Neurol Neurosurg Psychiatry 2012;83:620-8.

16 Boeve BF. Idiopathic REM sleep behaviour disorder in the development of Parkinson's disease. Lancet Neurol 2013;12:469-82.

17 Iranzo A, Valldeoriola F, Lomena F, et al. Serial dopamine transporter imaging of nigrostriatal function in patients with idiopathic rapid-eye-movement sleep behaviour disorder: a prospective study. Lancet Neurol 2011;10:797-805.

18 Malec JF, Brown AW, Leibson CL, et al. The mayo classification system for traumatic brain injury severity. J Neurotrauma 2007;24:1417-24.

19 Goetz CG, Fahn S, Martinez-Martin P, et al. Movement Disorder Societysponsored revision of the Unified Parkinson's Disease Rating Scale (MDS-UPDRS): Process, format, and clinimetric testing plan. Mov Disord 2007;22:41-7. 
20 Lapierre O, Montplaisir J. Polysomnographic features of REM sleep behavior disorder: development of a scoring method. Neurology 1992;42:1371-4.

21 Zijlmans J, Evans A, Fontes F, et al. [123I] FP-CIT spect study in vascular parkinsonism and Parkinson's disease. Mov Disord 2007;22:1278-85.

22 Jenkins PO, Mehta MA, Sharp DJ. Catecholamines and cognition after traumatic brain injury. Brain 2016;139:2345-71.

23 Jenkins PO, De Simoni S, Bourke NJ, et al. Stratifying drug treatment of cognitive impairments after traumatic brain injury using neuroimaging. Brain 2019;142:2367-79.

24 Norris EH, Giasson BI, Lee VM. Alpha-synuclein: normal function and role in neurodegenerative diseases. Curr Top Dev Biol 2004;60:17-54.

25 Goldman SM, Kamel F, Ross GW, et al. Head injury, alpha-synuclein Rep1, and Parkinson's disease. Ann Neurol 2012;71:40-8.

26 Uryu K, Chen XH, Martinez D, et al. Multiple proteins implicated in neurodegenerative diseases accumulate in axons after brain trauma in humans. Exp Neurol 2007;208:185-92.

27 Goldman SM, Tanner CM, Oakes D, et al. Head injury and Parkinson's disease risk in twins. Ann Neurol 2006;60:65-72.

28 Bauckneht M, Chincarini A, De Carli F, et al. Presynaptic dopaminergic neuroimaging in REM sleep behavior disorder: A systematic review and metaanalysis. Sleep Med Rev 2018;41:266-74.

29 Yan HQ, Kline AE, Ma X, et al. Traumatic brain injury reduces dopamine transporter protein expression in the rat frontal cortex. Neuroreport 2002;13:1899901. 
Jenkins 31

30 Shimada R, Abe K, Furutani R, et al. Changes in dopamine transporter expression in the midbrain following traumatic brain injury: an immunohistochemical and in situ hybridization study in a mouse model. Neurol Res 2014;36:239-46. 